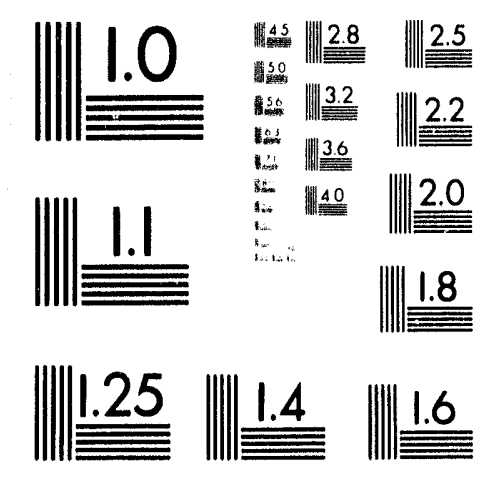



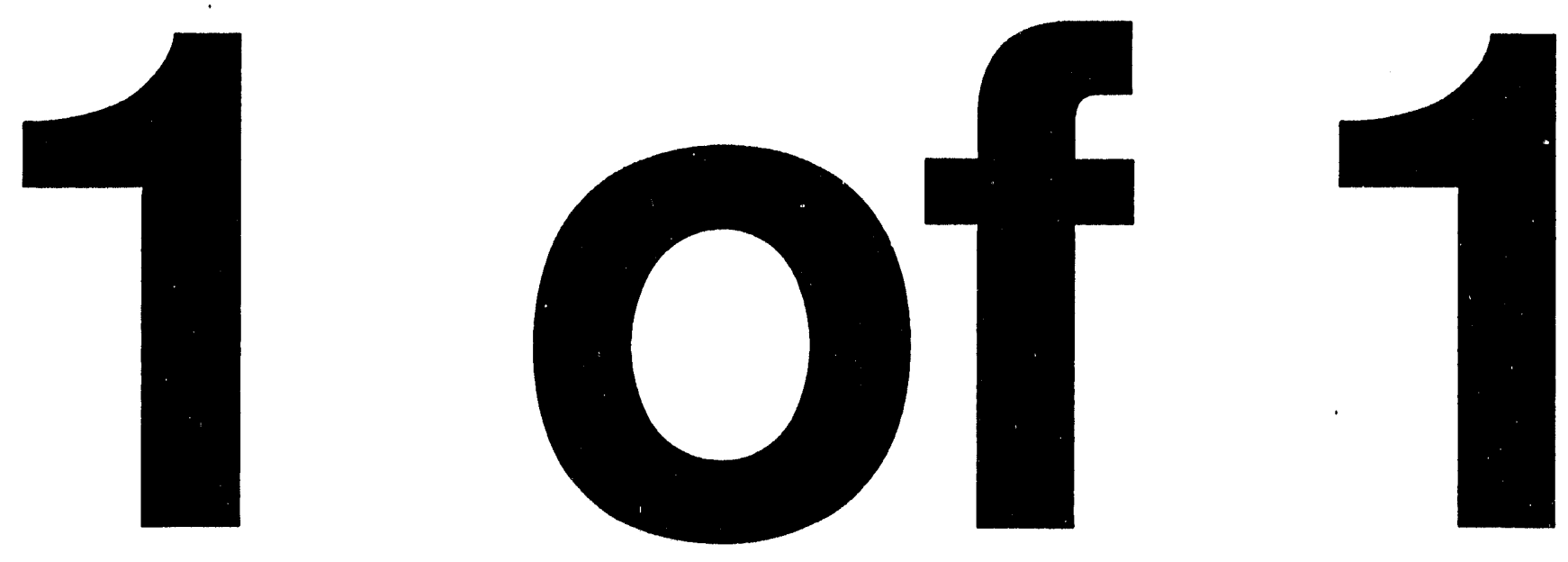
Engineering Physics and Mathematics Division

\section{MEASUREMENT AND ANALYSIS OF THE NITROGEN TOTAL AND DIFFERENTIAL SCATTERING CROSS SECTIONS IN THE RESONANCE REGION}

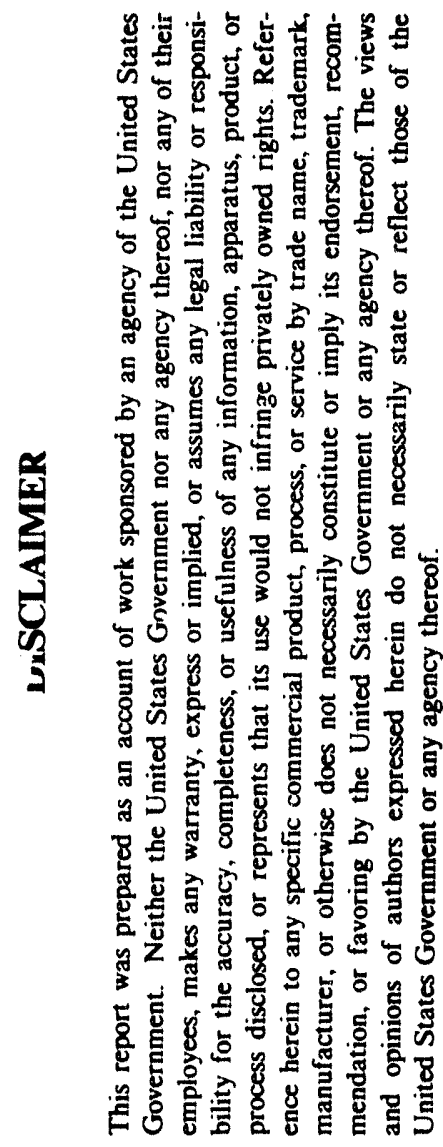

To be presented at the Eight ASTM-EURATOM

Cloyd O. Beasley, Jr.*

Nancy M. Larson*

J. A. Harvey*

Duane C. Larson*

Gerald M. Hale**

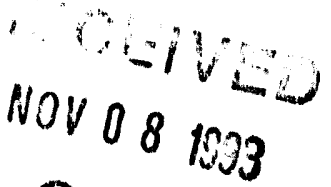

Symposium on Reactor Dosimetry

August 29 - September 3, 1993

vail, colorado

*Oak Ridge National Laboratory

Oak Ridge, Tennessee

**Theoretical Division

Los Alamos National Laboratory

Los Alamos, New Mexico

The submined monuscript hes been

guthored by contrector of the US

athored by inder contrect No. DE.

ACO5.840 21400 Accordingty, the U.S.

, Government reteins Accordingr. Thenchusive.

royelty-tree incense to publish of reproduce

the publiehed form of this contribution. or

allow others to do so, for U.S. Government

purposes."

Prepared by the Oak Ridge National Laboratory,

Oak Ridge, Tennessee 37831 managed by Martin Marietta Energy

Systems, Inc. for the U. S. Department of Energy under

contract DE-AC05-84OR21400. 
Cloyd O. Beasley, Jr.,' Nancy M. Larson, ${ }^{2}$ John A. Harvey, 3 Duane C. Larson, 4 and Gerald M. Hale

MEASUREMENT AND ANALYSIS OF THE NITROGEN TOTAL AND DIFFERENTIAL SCATTERING CROSS SECTIONS IN THE RESONANCE REGION

REFERENCE: Beasley, Cloyd O., Jr., Larson, Nancy M., Harvey, John A., Larson, Duane C., and Hale, Gerald M., "Measurements and Analysis of the Nitrogen Total and Differential Scattering Cross Sections in the Resonance Region," Reactor Dosimetry ASTM STP 1228, Harry Farrar IV, E. Parvin Lippincott, and John G. Williams, Eds., American Society for Testing and Materials, Philadelphia, 1994.

ABSTRACT: High-resolution neutron transmission and differential elastic scattering measurements have been made on samples of nitrogen from $0.5 \mathrm{eV}$ to $10 \mathrm{MeV}$ at the Oak Ridge Electron Linear Accelerator (ORELA). For the transmission measurement several different detectors were used at various flight path lengths, with high-purity

nitrogen gas samples. The data have been corrected for the dead time of the time digitizer and for several small backgrounds. For the scattering measurements six detectors were placed at various angles inside an evacuated scattering

'Operations Manager, Oak Ridge National Laboratory, Engineering Physics and Mathematics Division, MS-6361, P. O. Box 2008, Oak Ridge, TN 37831. MS -6356 .

${ }^{2}$ Ibid., Research Staff, Computing Applications Division,

${ }^{3}$ Ibid., Director, Oak Ridge Electron Linear Accelerator (ORELA), Engineering Physics and Mathematics Division, MS6356.

"Ibid., Section Leader, Nuclear Data Measurement and Evaluation, Engineering Physics and Mathematics Division, MS6354.

${ }^{5}$ Theoretical Division, Los Alamos National Laboratory, Los Alamos, NM 87545 . 
chamber at 200 meters from the neutron producing target. The sample was silicon nitride held in a thin graphite container. Scattering from carbon and silicon samples was also measured to correct for these materials and calibrate the relative efficiencies of the scattering detectors. The data have been corrected for dead time and for a constant background. A preliminary analysis has been completed to obtain parameters of the resonances up to $4.8 \mathrm{MeV}$.

KEYWORDS: ${ }^{14} \mathrm{~N}$ high-resolution transmission and scattering measurements, R-matrix analysis, resonance parameters.

\section{INTRODUCTION}

Efforts to reproduce the measured activity induced by low energy neutrons in Cobalt-60, Chlorine-36, and Europium152,154 as a function of distance from ground zero at 500 to 2000 meters resulting from the Hiroshima nuclear explosion have been unsuccessful. Improved neutron cross sections of nitrogen (N) and oxygen (O) for neutrons from low energies up to the energy of the source are needed to calculate the neutron transport in air at these large distances. A recent neutron cross section evaluation for nitrogen (ENDF/B-VI Mod 0) by Young, Hale and Chadwick [1] was completed in 1990. Some experimental data below $500 \mathrm{keV}$ neutron energy were over 30 years old. This evaluation work resulted in a request for new neutron cross section measurements for nitrogen covering a wide energy range. This paper describes the high-resolution neutron total and differential elastic scattering measurements from $0.5 \mathrm{eV}$ to $10 \mathrm{MeV}$ which were made at the ORELA to satisfy the request. Using this new total cross section data, Hale [2] did a new R-Matrix analysis of the nitrogen resonance region and found a better fit if spins and parities of some resonances were changed from those used in ENDF/B-VI Mod 0 . These results were used in an update [1] of the nitrogen evaluation (Mod 1) issued in June, 1992. Since differential scattering data are more sensitive to parities of resonances, the present paper reports an analysis of the new ORELA scattering data to verify the spins and parities found by Hale in his analysis for Mod 1 .

Details of the transmission measurement have been reported previously and are only briefly reviewed here. In the present work, total and differential elastic data were analyzed with the R-matrix code SAMMY [3] to obtain resonance parameters consistent with both types of data. 
EXPERIMENTAL TRANSMISSION MEASUREMENTS

High-Resolution Measurements from 0.1 to $20 \mathrm{MeV}$

For the high-resolution transmission measurements in this energy region, unmoderated neutrons directly from the ORELA tantalum target were produced by $5-$ nsec wide $150-\mathrm{MeV}$ electron pulses at a repetition rate of 800 pps. Details of this measurement are reported in an earlier paper. [4]

Measurements from $0.5 \mathrm{eV}$ to $200 \mathrm{keV}$

For the transmission measurements in this energy region the collimation was changed so that only neutrons from the water moderator of the ORELA target could reach the neutron detector. The burst width was $30 \mathrm{nsec}$ with a repetition rate of 80 pps. Additional details are reported in the earlier paper. [ㄴ]

\section{EXPERIMENTAL DIFFERENTIAL ELASTIC SCATTERING MEASUREMENTS}

High-resolution scattering measurements were done at the 200-m flight-path station. A collimator allowed both moderated and unmoderated neutrons to reach the sample.

providing a nitrogen sample in a form suitable for scattering measurements is a difficult problem; we finally settled on silicon nitride. The sample consisted of $57.80 \mathrm{~g}$ of silicon nitride $\left(\mathrm{Si}_{3} \mathrm{~N}_{4}\right)$ powder contained in a $8.4 \mathrm{~g}$ hollow graphite cylinder with a $0.05 \mathrm{~cm}$ bottom $(7.0 \mathrm{~cm}$ high, $3.81 \mathrm{~cm}$ outside diameter, and $3.70 \mathrm{~cm}$ inside diameter). The cylinder was suspended at the center of a 183-cm-diam scattering chamber, which was evacuated and isolated from the flight-path beam tube by means of a $0.025-\mathrm{cm}$ Mylar entrance window. Measurements were also made with a silicon (Si) sample $6.17 \mathrm{~cm}$ diameter and $0.58 \mathrm{~cm}$ thick mounted at a $631^{\circ}$ angle from the direction of the neutron beam.

The scattering data were obtained using a $0.3-\mathrm{g} / \mathrm{cm}^{2}{ }^{10} \mathrm{~B}$ filter to eliminate low-energy neutrons associated with the previous burst and with two filters of Uranium-238 to reduce the intensity of the gamma flash from the target. One filter $(0.73 \mathrm{~cm}$ thick) covered the whole collimator; the other $(2.5 \mathrm{~cm}$ thick) shadowed only the tantalum part of the target. The measurements covered the energy region from approximately $10 \mathrm{keV}$ to $10 \mathrm{MeV}$. The electron beam burst width was 9 nsec.

Six neutron detectors were located $19.1 \mathrm{~cm}$ from the center of the chamber at laboratory angles of $36.1^{\circ}, 51.8^{\circ}$, $86.0^{\circ}, 116.5^{\circ}, 137.2^{\circ}$, and $158.5^{\circ}$ from the direction of the incident neutron beam. Each neutron detector consisted of a 7.62-cm-long by 4.32-cm-diam cylinder of NE-110, which was viewed at each end by RCA 8850 photomultiplier tubes. Additional details of the experimental arrangement for these 
scattering measurements can be found in Ref. [ [ ] ; information on the calculated efficiencies for the twophototube detectors is given in Ref. [ㅎ].

Data were also taken with one of the detectors placed in the incident beam to measure the product of the fiux and detector efficiency as a function of neutron energy. Scattering measurements using a graphite sample were also made to determine the relative efficiencies of the other five detectors.

All spectra were normalized by means of a neutron monitor detector. After correcting for dead time and constant room background, the scattering spectra were divided by the spectrum from the in-beam detector to remove effects of the energy dependence of the incident flux and detector efficiency. The data were not corrected for multiple scattering in the sample (this was done as part of the R-matrix analysis), and have an uncertainty of $\approx 10 \%$ for the relative differential scattering cross section.

\section{R-MATRIX ANALYSES OF THE CROSS-SECTIONS}

Values of resonance parameters (resonance energy and widths) and assignment of quantum numbers (spin $\mathrm{J}$, and orbital angular momentum $l$ and corresponding parity $\pi$ ) may be determined or confirmed through combined analyses of the total cross-section and differential elastic scattering cross-section data. The differential scattering data are particularly useful in determining values for $l$ (and $\pi$ ). The analysis is complicated, however, because of a number of experimental impediments:

(a) The target used was $\mathrm{Si}_{3} \mathrm{~N}_{4}$. But silicon itself has many resonances, and accurate determination of the silicon resonances is necessary to properly analyze the nitrogen resonances. Even so, existence of a silicon resonance at an inauspicious energy can result in masking of the desired nitrogen scattering behavior.

(b) At higher energies, not only do the peak crosssections of resonances decrease, but the differential scattering becomes less sensitive to differences in quantum numbers, $l$ in particular.

(c) The target must be a finite size in order to obtain sufficient scattering counts; but the finite size presents resolution problems, as well as absorption of scattered particles. This phenomenon is best seen in forward scattering, where the experimental peak height is often lower than predicted, although acceptable energy dependence for a given set of quantum numbers can be obtained at other angles.

Our work to provide the best possible parametric assignments for all nitrogen resonances is on-going; preliminary results are reported here. The current "best 
fit" to the total cross section data is shown in Figure 1 , for energies between the lowest resonance (at $433 \mathrm{keV}$ ) and $4.8 \mathrm{MeV}$.
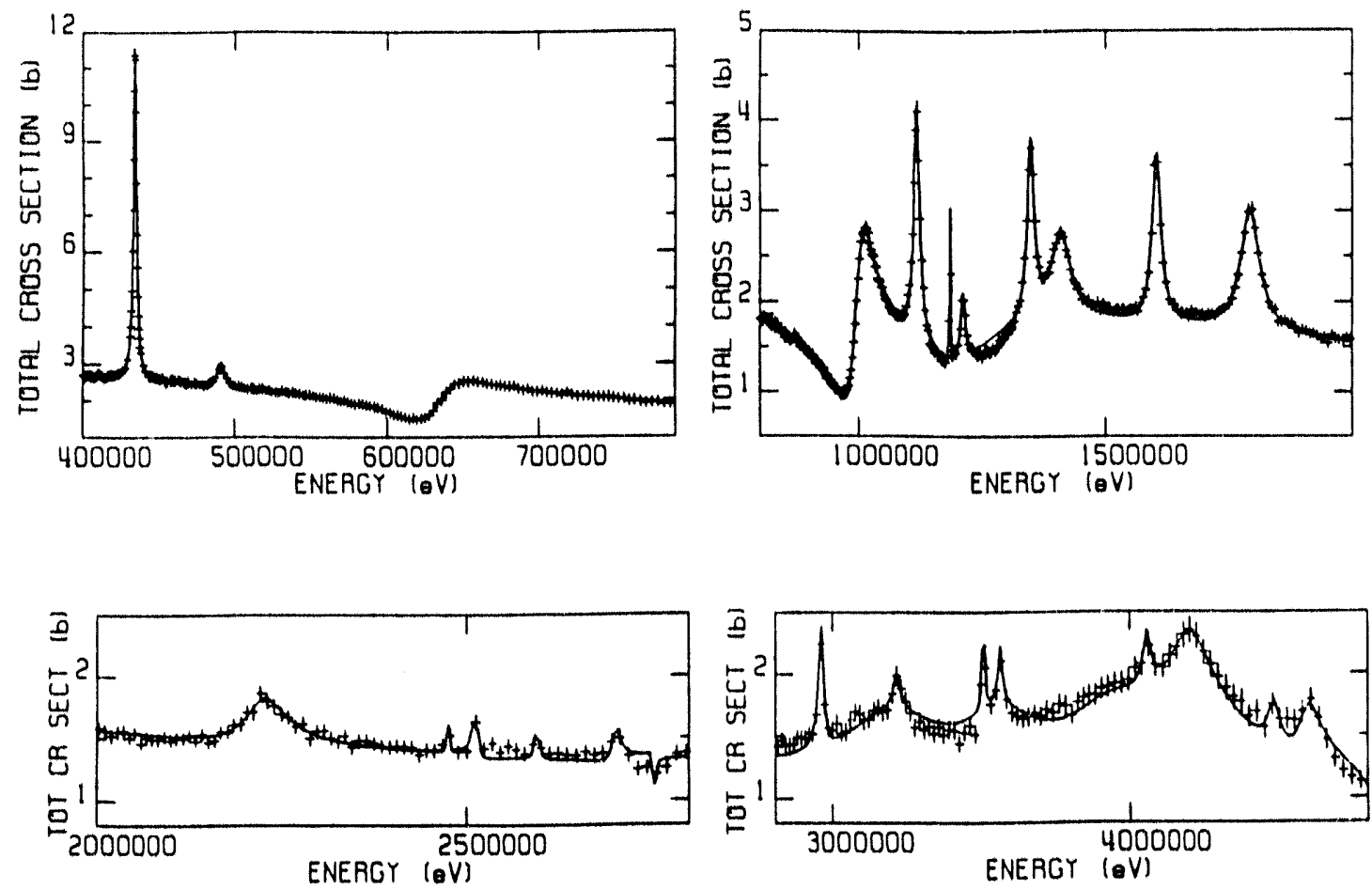

Fig. 1--Total cross section from the high-resolution ORELA transmission measurement. The solid curve shows our preliminary R-matrix fit.

We now consider the spin/parity assignments for the individual resonances from $433 \mathrm{keV}$ to $2.5 \mathrm{MeV}$.

Prior to ENDF/B-VI Mod 1, the spin assignment for the $433-\mathrm{keV}$ resonance had been taken to be $3 / 2$. After that time, analysis of our high-resolution measurements [ 3 ] of the total cross section determined that $J$ must be $7 / 2$, and that value was used in the modified ENDF/B-VI (Mod 1) analysis by Hale [1]. The differential scattering data provide confirmation of this assignment, and clearly indicate $l=2$ (rather than $l=3$ ) is the correct assignment, as shown in Fig. 2 . 

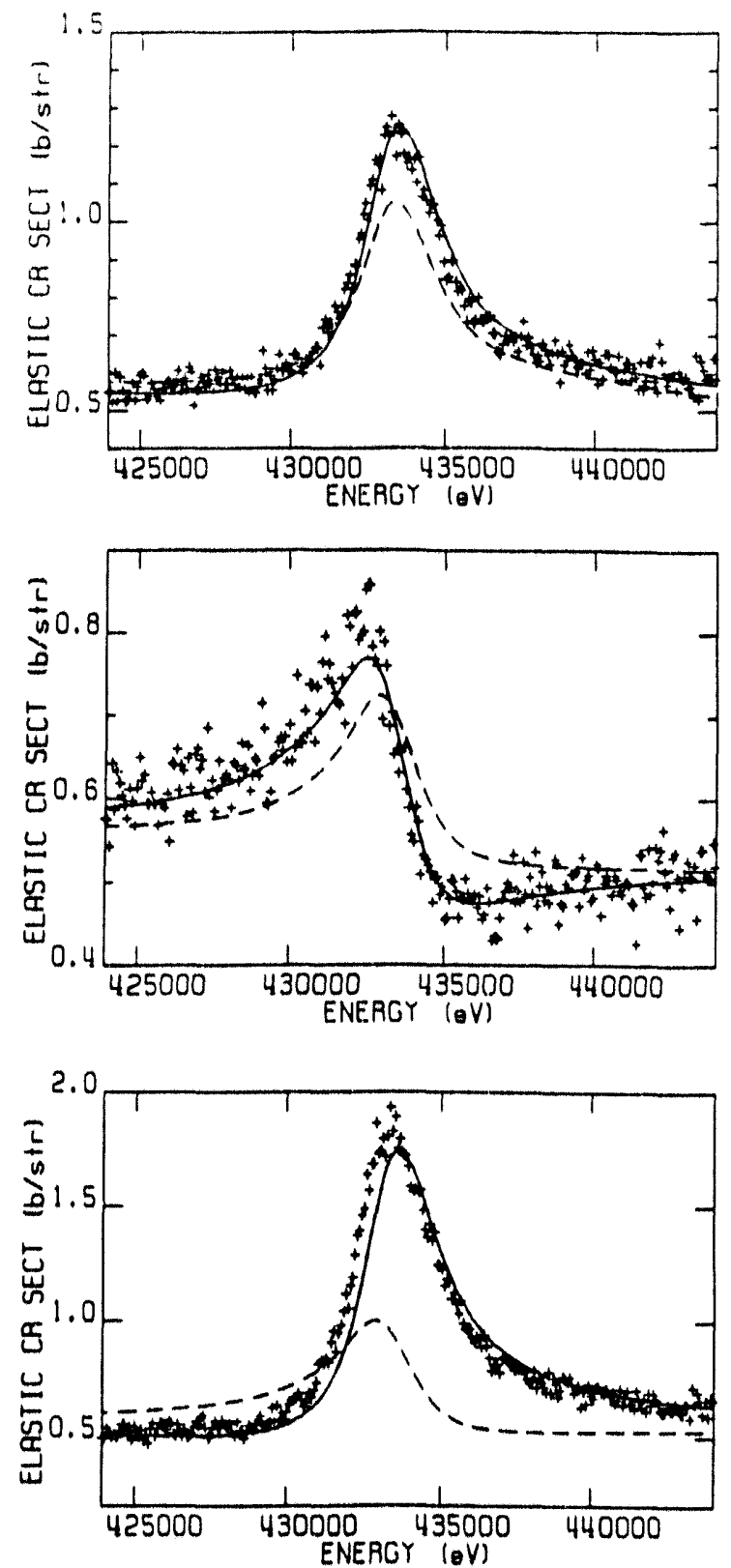

Fig. 2--Differential elastic scattering cross section for the $433-\mathrm{keV}$ resonance of ${ }^{14} \mathrm{~N}$, at laboratory angles of 36.6 , 86.0 , and 158.5 degrees respectively. The solid curve represents the $\ell=2$ assignment, and the dashed $l=3$.

[Note: An approximation was used in determining the experimental energies of the scattered neutrons from the time-of-flight information; the approximation appears to break down at backward angles. Thus the structure in the calculation appears to be at higher energy than the corresponding structure in the data. Since this and other 
difficulties with the differential elastic scattering data have yet to be fully considered, thus far these data are used only to determine the quantum numbers and not for precise fitting of resonance parameters.]

The $491-\mathrm{keV}$ resonance is too small to obtain a clear spin assignment from the differantial scattering data. The data, however, are not inconsistent with the previous assignment of $1 / 2^{\circ}$ for $\mathrm{J}^{n}$.

The $639-\mathrm{keV}$ and $997-\mathrm{keV}$ resonances are both definite swaves $(l=0)$, the first with $J=1 / 2$ and the second $\mathrm{J}=3 / 2$.

The resonance at $1.116 \mathrm{MeV}$ had been assigned $\mathrm{J}^{\pi}=5 / 2$ [2]. Figure 3 shows the differential elastic data for this resonance, compared with both positive and negative parity theoretical curves, confirming that the negative-parity assignment is reasonable.
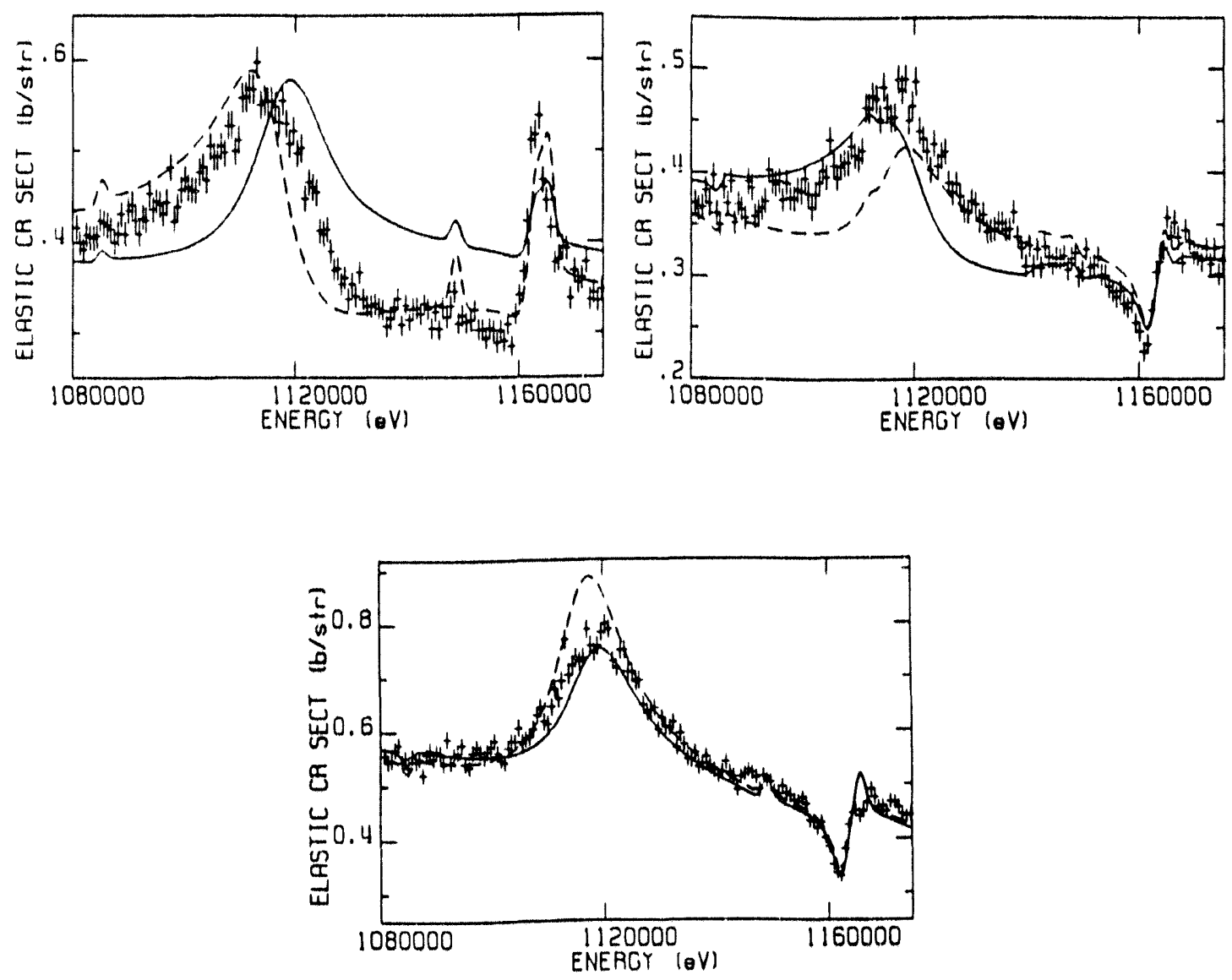

Fig. 3--Differential elastic scattering cross section for the $1.116-\mathrm{MeV}$ resonance, at laboratory angles $36.6,86.0$, and 158.5 degrees. The dashed curve represents the positive-parity assignment, and the solid the negativeparity. 
The previous assignment for the $1.185 \mathrm{MeV}$ resonance was $J=5 / 2$. Comparison of theoretical calculations with the differential elastic scattering data is shown in Fig. 4. Based on these calculations we tentatively assign negative parity to this resonance.
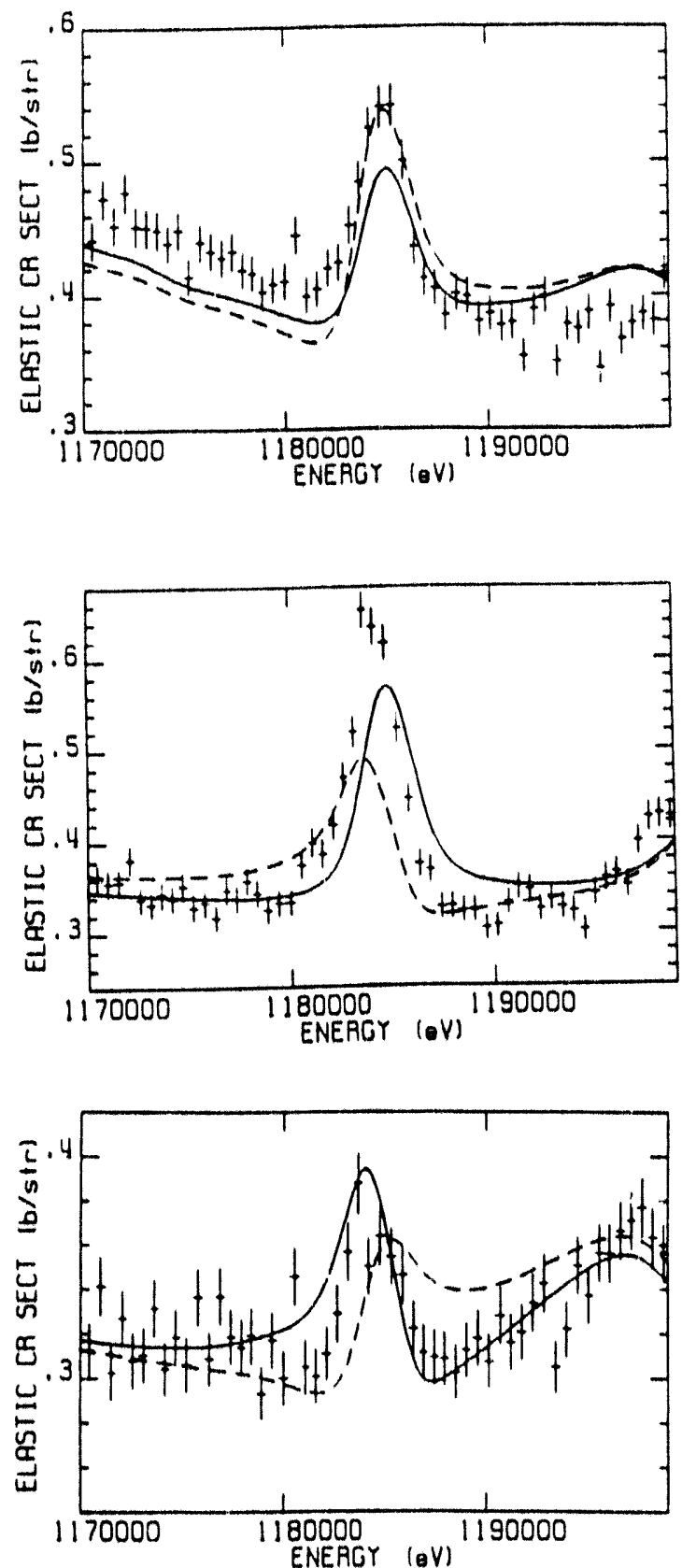

Fig. 4--Differential elastic scattering cross section for the $1.185-\mathrm{MeV}$ resonance, at laboratory angles $36.6,86.0$, and 158.5 degrees. The dashed curve represents the positive-parity assignment, and the solid the negativeparity. 
The $1.21 \mathrm{MeV}$ resonance was assigned $J^{n}=1 / 2^{\circ}$. The differential elastic data provide no definitive information about this resonance, primarily because of the presence of nearby silicon resonances.

A broad $l=0$ state underlies this region; Hale [2] determined this state to be $\mathrm{J}^{\pi}=3 / 2^{+}$at energy $1.255 \mathrm{MeV}$. The $1.35-\mathrm{MeV}$ and $1.41-\mathrm{MeV}$ resonances have been assigned $\mathrm{J}^{\pi}=5 / 2^{+}$and $3 / 2^{\circ}$ respectively. Our analysis of the differential elastic data confirms these assignments. Resonances at 1.60 and $1.78 \mathrm{MeV}$ were previously assigned $\mathrm{J}^{\pi}=5 / 2^{\circ}$ and $5 / 2^{+}$respectively. The differential scattering data are inconclusive on the parities.

The resonance at $2.220 \mathrm{MeV}$ had been assigned $\mathrm{J}^{n}=3 / 2{ }^{\circ}$. Calculations of the differential elastic scattering cross section for this resonance show almost no distinction between the positive and negative parity options; neither is inconsistent with the data. Data from other reactions had been used to determine that the parity is negative [2].

\section{CONCLUSION}

Total and differential elastic scattering data for nitrogen have been obtained at ORELA and analyzed with the R-matrix formalism. The scattering data have provided essential information to aid in determining the orbital angular momentum $l$ values for resonances. The data have confirmed some earlier results and provided new $l$ values for other resonances. The large resonance at $433 \mathrm{keV}$ has been shown to have $l=2$.

\section{ACKNOWLEDGMENTS}

This research was sponsored by the office of Energy Research, Division of Nuclear Physics, U.S. Department of Energy, under contract No. DE-AC05-840R21400 with Martin Marietta Energy Systems, Inc., and under Interagency Agreement No. 0046-C083-A1 with the Defense Nuclear Agency.

\section{REFERENCES}

[1] P. Young, G. Hale, and M. Chadwick, ENDF/B-VI Mod 0 evaluation for ${ }^{14} \mathrm{~N}$, MAT 725 (June 1990), and ENDF/B-VI Mod 1 (June 1992).

[2] Use of R-Mateix Theory in Light Element Evaluations, Gerald M. Hale, presented at the symposium of Nuclear Data Evaluation Methodology, Brookhaven National Laboratory, Upton, Long Island, New York, October 1992; to be published. 
[3] Updated Users' Guide for SAMMY: Multilevel B-Matrix Fits to Neutron Data Using Bayes' Equations, N. M. Larson, ORNL/TM-9179, August 1984; ORNL/TM-9179/R1, JulY 1985; ORNL/TM-9179/R2, June 1989.

[4] J. A. Harvey, N. W. Hill, N. M. Larson, and D. C. Larson, "Measurement of the Nitrogen Total Cross Section from 0.5 $\mathrm{eV}$ to $50 \mathrm{MeV}$, and Analysis of the 433-keV Resonance," pp. 729-731 in proceedings of the Conference on Nuclear Data for science and Technology, Forschungszentrum Jülich, Germany, May 13-12, 1991, ed. S. M Qaim, springer-Verlag, Berlin, 1992.

[5] D. J. Horen, C. H. Johnson, J. L. Fowler, A. D. Mackellar, and B. Castel, Phys. Rev. C 34, 429 (1986).

[6] N. W. Hill, J. A. Harvey, D. J. Horen, G. L. Morgan, and R. R. Winters, IEEE Trans, Nucl, SCi, NS-32, 367 (1985). 

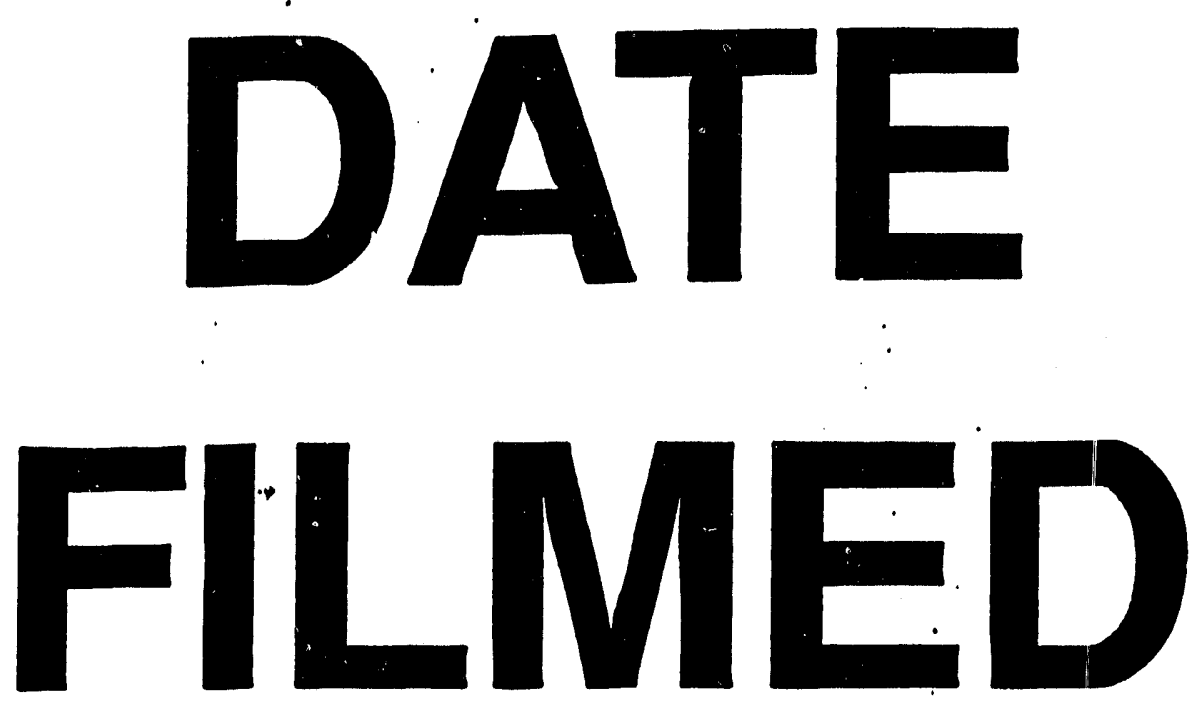

$12 / 29 / 93$
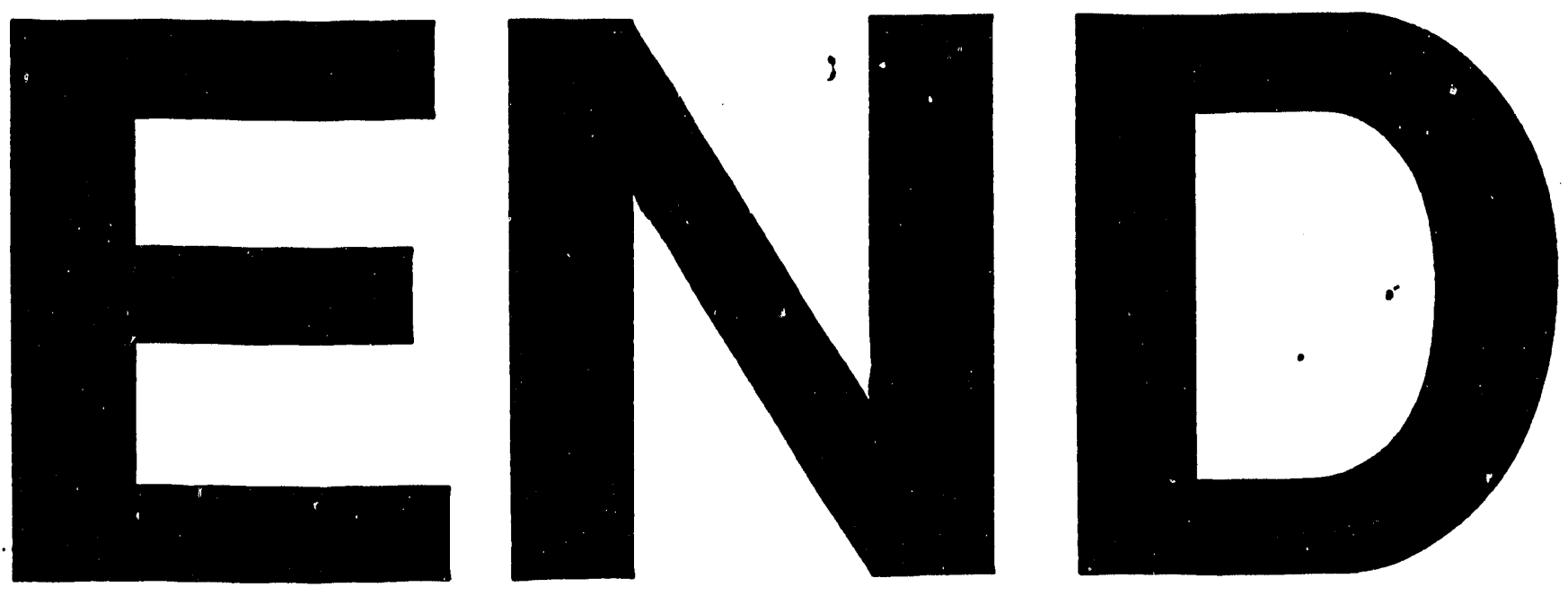


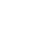

